Pub. Mat. UAB

№ 12 Juny 1979

\title{
ALGUNS ASPECTES ACTUALS DE LÖGICA ALGEBRAICA
}

\section{Josep Pla}

Facultat de Matemàtiques.

Universitat de Barcelona.

L' any 1955 Paul $k$. HAlos treu a "Compositio Mathematicae, vol.12" 1'article "slgebraic Logic" en el que dóna una formalització algebraica del concepte cle deducció. A I'entorn dels anys seixanta l'escola dintonio NONTEIro dóna una ma teixa presentació algebraica de 13 loggica però, en lloc de centrar la seva atenció en la lòsica booleana, debilita la questió al substrat positiu de la lògica.

La idea que hi ha al dessota d'aquesta presentació és la següent :

si pensem en el càlcul de proposicions construit sobre un conjunt no buit $x$ de lietres com en l'àlgebra lliure $i^{2}(x)$ sobre $\mathrm{X}$ d'operacions $\neg \mathrm{i} \rightarrow$ (monària, la jrimera; binària, la segona) tenim que, si $x \in x$, aleshores $x i \neg \neg x$ són dues expressions diferents en $P(X)$. Aleshores cal introduir - i s'introdueix - una relació d'equivalència en $P(X)$ que identifiqui, d'acord amb el que nosaltres es xerem tlel càlcul de sroposicions, certes fórmules.

El camí sintàctic consisteix en clonar el concepte de demostració : s'aģafen certes expressions distingides en $\mathrm{P}(\mathrm{X})$ : $\mathcal{R}_{1}=\{\mathrm{P} \rightarrow(q \rightarrow p)$, on $\mathrm{p}, \mathrm{q} \in \mathrm{P}(\mathrm{x})\} ;$

$$
\begin{aligned}
& \left\llcorner A_{2}=\{(p \rightarrow(q \rightarrow r)) \rightarrow((p \rightarrow \eta) \rightarrow(p \rightarrow r)) \text {, on } p, q, r \in P(x)\}\right. \text {; } \\
& \mathrm{A}_{3}=\left\{\neg \neg \mathrm{p} \rightarrow \mathrm{p} \text {, on } \mathrm{g} \in \mathrm{P}^{\mathrm{P}}(\mathrm{X})\right\} ;
\end{aligned}
$$

i una regla de deducció, anomenada "modus ponens" : M.P.:

$$
R=\{(p, p \rightarrow q, q): p, q \in P(x)\} \circlearrowleft\{(p \rightarrow a, p, q): p, q \in a f x)\} .
$$


J'anomena teorema tota expressió $\mathfrak{f} \in \mathfrak{i}^{2}(x)$ tal que :

existeix una successió Einjta 4 'expressions ${ }^{1}, \ldots,{ }_{n}$, $p_{i} \in P(x) \quad(i=1, \ldots, n) \quad a m b ?_{n}=p$ tal que

1. $\stackrel{9}{i}_{i} \in A_{1} \backsim A_{2} w \mathrm{AH}_{3}$, obé

2. existeixen $p_{j}, p_{k}(j, k<i) i\left(p_{j}, p_{k}, p_{i}\right) \in k$.

Diem que una expressió és un teorema usant el símbol : b. $\supset$. dquesta idea es pot generalitzar a qualsevol conjunt $A \subseteq P(X)$ $i$ introduir els $A-t e o r e m e s ~(A \longmapsto p)$. IIom consiclera els axiomes $A(A)=A_{1} \cup A_{2} \cup A_{3} \cup A$.

Dien que $p \equiv q$ si, $i$ només si, $\vdash \rho \rightarrow q \quad i \mapsto q \rightarrow p$. Es una relació d'equivalència $i$ resulta que $P(x) / \equiv$ es una àlgebra de Boole : 1'àlgebra de Tarski-Lidenbaum.

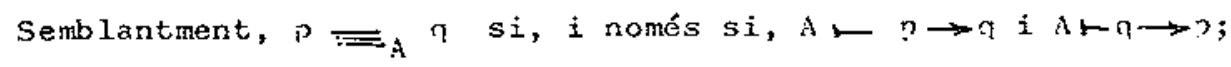
resulta que $P(x) / \rightleftharpoons_{\lambda}$ es també una àlgebra de Boole.

És aquest ordre d'idees el que recull l'escola d'A. MONTEIRO (A. MONTEIRO [1960]; [1971]; Antonio DIEGO [1965]; [1966]) quan defineix les àlgebres de llilbert i les preàlgebres de Hilbert.

Hom diu que una terna $(A, \ldots, D)$ és una preàlgebra de Hilbert si, i només si,

$\mathrm{PH}_{1}$. És una operació binària en A; $\mathrm{PH}_{2}, \quad \mathrm{D} \subseteq \mathrm{A}$;

$\mathrm{PH}_{3} \cdot \quad x \cdot(y \cdot x) \in D$, per cada parell $x, y \in A$; $\mathrm{PH}_{4} \cdot(x \cdot(y, z)) \cdot((x \cdot y) \cdot(x, z)) \in D$, per $x, y, z \in \Lambda$; $\mathrm{PH}_{5}$ M.P.: $\mathrm{si} x \in \mathrm{D}$ i $x \cdot y \in D$, aleshores $y \in D$.

És important d'observar que la relació en A, $x \epsilon_{D} y \quad s i, i$ només si, $x, y \in D$

és una relació de preordre - tal com passava en $P(x)$ $i$, per tant, tenim una relació d'equivalència $\bar{D}_{\mathrm{D}}$. 
L'àlgebra quocient, ja que $\equiv_{D}$ és compatible amb., dóna

$$
\left(A / \equiv{ }_{D}, \ldots, D\right) \text {, }
$$

on . és una operació en $A / \equiv_{D}, D \in M / \equiv_{D} i$ és màxim en $\left(A / \equiv D, \ldots, \leqslant_{D}\right)$.

Aruest tipus d'estructures s'anomenen àlgebres de Hilbert - i són la rèplica de les algebres de Boole quocient - i es caracteritzen :

Una terna $(A, \ldots, u)$, on . és una operació binària en $A$ i $u \in A$, és una àlgebra de Hilbert si, i només si, $\mathrm{AH}_{1} \cdot$ La relació $x \leq y \mathrm{si}$, i només $s i, x, y=u$ és una relació d'ordre;

$\mathrm{AH}_{2} \cdot \quad \mathrm{x} \cdot \mathrm{u}=\mathrm{u} ; \mathrm{u} \cdot \mathrm{x}=\mathrm{x}$;

$\mathrm{AH}_{3} \cdot \quad x \cdot(\mathrm{y}, \mathrm{z})=(\mathrm{x}, \mathrm{y}) \cdot(\mathrm{x}, \mathrm{z})$.

Aquesta definició es troba en Francesc d'Assis SALES [1971].

D'aquesta manera hem introduit de forma natural el conceste d'algebra de Hilbert, que podem pensar com l'ent algebraic més idoni per a descriure la lògica proposicional positiva.

En una àlgebra de Hilbert $(A, \ldots, U)$ els sistemes deductius $D$ són els subconjunts $D \subseteq A$ tals que

$$
\begin{array}{ll}
\mathrm{SD}_{1} \cdot & x \in \mathrm{D} ; \\
\mathrm{SD}_{2} . & x \cdot y \in \mathrm{D} \quad \mathrm{i} \quad x \in D, \text { aleshores } y \in D .
\end{array}
$$

Hom pot veure que $\theta=\{D$ : D és un s.d. de $(A, \ldots, u)\}$ és un sistema clausura - una col.lecció de parts de A tancada per interseccions arbitràries - que verifica : és finitari; el

teorema de 1a deducció de Tarski.

Finitari: $x \in D$ si, $i$ només si, existeix una col.lecció finita $x_{1}, \ldots, x_{n} d^{t e l e m e n t s ~ d e ~} D i x \in D\left(x_{1}, \ldots, x_{n}\right)$ - s.d. engendrat per $x_{1}, \ldots, x_{n}$. 
Teorema de la deduccíb: $x \in D(D, y)$ si, $i$ només si, $y \cdot x \in D$, on $D$ es un s.d., $x, y \in A$ arbitraris $i \mathrm{D}(D, y)$ és el s.d. engendrat per $D i$ per $y$.

En aquest ordre d'idees s'insereixen els treballs de Helena RASIOWA $i$ ROMan SIKORSKI, Veure H. Rasiowa [1970], [1974]. Els sistemes deductius irreductibles serveixen per a donar una representació de Stone de les àlgebres de Hilbert.

Conve indicar - tan sols de passada - que les topologies que hom troba amb aquest tipus de representacions són topologies poc desitjables des d'un punt de vista topologic $i$ de l'analisi.

Un altre ordre d'idees (cf. Rasiowa, op.cit.) que hom pot plantejar-se un cop summergit en aquest àmbit és el que ens planteja la segflent questio :

a partir d'una operació . de Hilbert, és possible d'alguna manera introduir operacions reticulars en el conjunt ordenat $(A, \ldots, u)$ ? (Pensem que en les àlgebres de Boole, gràcies a la complementació, podem, a partir de la implicacio, definir les operacions reticulars; ara però no disposem pas de negació.)

Les àlgebres de Abbott-Sales (Abbott [1970]; F.A. Sales [1974]; J,Pla [1977]) permeten definir, a partir de., un suprem per a cada dos elements, $\mathrm{Cal}$ indicar que aquestes àlgebres tenen una particularitat curiosa $i$ ss: si tenen element minim són algebres de Boole; $i$ els seus sistemes deductius irreductibles, maximals i primers coincideixen.

Diem qué és una algebra de Abbott-Sales : és una àlgebra de Hilbert $(A, ., u)$ tal que

$$
(x \cdot y) \cdot y=(y \cdot x) \cdot x \quad \text { (caracteritzacio de Sales [1974]). }
$$

A leshores

$$
x \vee y=(x \cdot y) \cdot y \text { és un suprem en }(A, \leqslant) .
$$


Si agafem dientrada un reticle (A, $\wedge, V$, $u$ ) i una operacib binària. en A tal que $(A, \ldots, H)$ sigui una àlgebra de Hilbert, obtenim els anomenats reticles de Hilbert (cf. Sales I1974]; J.PIa $[1977\} ;\{1978\})$ si l'ordre del reticle i t'ordre induIt per . coincideixen. (Si en lloc d'un reticle prenem un suprareticle obtenim els suprareticles de Hilbert.)

En aquest context hem pogut donar un tractament unitari de les algebres de Abbott-Sales, de Heyting, de Boole. Totes elles son generalitzacions reticulars del substrat eminentment lògic de les àlgebres de Hilbert.

Hom demostra que els filtres de reticle de $(A, \wedge, V, u)$ són sistemes deductius, que els sistemes deductius orimers caincideixen amb els sistemes deductius irreductibles i s'obté, amb certes limitacions, una representació de Stone d'aquestes noves estructures lògic-algebraiques.

Una questio que hom pot plantejas encara en una àlgebra de Hilbert (resp. en un reticle o en un suprareticle de Hilbert) és la questió de les negacions. Això porta a introduir el concepte algebraic de negacio en un conjunt ordenat (resp. en un reticle, en un suprareticle).

Si $(A, \leqslant)$ es un conjunt ordenat (cf. G.BODIOU [1964]), tota aplicacio

$$
\text { こ : A } \longrightarrow A
$$

tal que $N_{1}, \quad x \leqslant y$ implica $\tau y \leqslant \tau x ; N_{2} \cdot x \leqslant \tau^{2} x$ s'anomena negacib.

Una negacio forta és tota negació que verifica $\tau^{2} x=x$, per tot $x \in A$. Si A és un reticle, $\tau$ es una negació reticular o de Morgan si és negacio $i$, a més : $N_{3}$. $\tau(x \wedge y)=$ $\tau \mathrm{x} \vee \tau \mathrm{y} ; \tau(\mathrm{x} \vee \mathrm{y})=\tau \mathrm{x} \wedge \mathrm{y}$

(No cal pas que sigui forta, si bé tota negacib forta és reticular, si esta definida en un reticle). (Sobre questions 
generals de les negacions en reticles complets, cf. F. ESTEVA [1974]).

Si portem aquestes idees a les àlgebres de Hilbert veiem que, per a tot a $\in A, I^{\prime a p l i c a c i b}$

$$
\begin{aligned}
\tau_{a}: A & \longrightarrow A \\
x & \longmapsto \tau_{a}(x)=x \cdot a
\end{aligned}
$$

és una negació. Si $\tau_{a}$ és forta, A té mínim $i \mathrm{a}=$ min (A).

De forma semblant hom pot analitzar en els reticles de Hilbert cóm són les negacions reticulars. Si be Haskel1 CuRRY r1952 1 realitza un andisi en açuest sentit no coneixem fins avii cap treball exhaustiu en aquesta lfnia $i$, per tant, constitueix una questio àmplia oberta.

Continuant en la semàntica que s'inspira en la lògica clàssica, podem 1 ligar les negacions amb els sistenes deductius via el concepte de consistència. .

Fóra interessant efectuar, també en aquest sentit, un estudi exhaustiu que, avui ger avui, no s'ha pas fet.

Una altra idea desenvolupada per P.R. Halmos (op.cit.) i independentment per L.HENKIN-J.D.MONK-A.TARSKI [1971] i L.Kenkin-A.Tarski [1960), (1961]), consisteix en introduir en les algebres de Boole operadors que, d'alguna manera, recullin la idea dels quantificadors. Així Halmos construeix les àlgebres monàdiques $i \mathrm{H}-\mathrm{M}-\mathrm{T}$ construeixen les àlgebres cilíndriques. A.Monteiro recuIl aquestes idees en A.Monteiro ([1957]; [1960]; [1967]; [1974]) i les aplica a estructures més febles com són les àlgebres de Heyting. No coneixem, però, cap treball de reticles de Hilbert monadics, ni tampoc dialgebres de Hilbert monadiques. La dificultat rau en el fet que per introduir els citats operadors hom utilitza les propietats reticulars i no coneixem cap treball en que $s^{i}$ introdueixin via 1 'operacib de Hilbert. (que és l'operacíb de la logica). 
Creiem que, en les àlgebres de Abbott-jales, podriem perfectament introduir operadors existencials o universals i obtindre àlgebres de Abbott-3ales monàdiques. Hi estem treballant .

Donem la idea de Halmos d'àlgebra monàdica. Sigui

$(A, \wedge, \vee, \neg ; 0, u)$ una àlgebra de Boole $i$ sigui

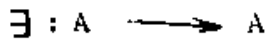

tal que

$E_{1} \cdot \exists 0=0 ; E_{2} . \quad x \leq \exists x ; \quad E_{3} \cdot \exists(x \vee \exists y)=\exists x \vee \exists y$. En aquestes àlgebres els sistemes deductius monadics són els sistemes deductius tals que, si $\exists x \in D$, aleshores $x \in D$.

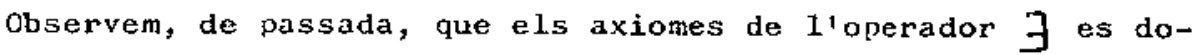
nen a traves de les operacions reticulars i d'ordre.

Convé dir, a fi d'ésser rigurosos, que, si bé les àlgebres cilindriques intenten algebritzar el calcul de predicats $i$ fan intervenir variables, les àlgebres monàdiques algebritzen les lògiques modals.

Una segona via per a donar el concepte de lògica algebraica fou introduida l'any 1930 per A.Tarski (cF.A.Tarski [1930])

i la idea expressada en acjuest trebal1 la podem descriure semànticament de la forma segllent :

"Donar una lògica equival a donar el conjunt

de consequlències de cada conjunt".

Formalment una lògica abstracta és una parella $(A, C)$, on $A \neq \emptyset$ i $C: \Phi(\mathrm{A})$ $P(A)$

verifica :

$$
\begin{aligned}
& C_{1} \text {. Per tot } x \subseteq A, \quad x \subseteq C(x) \text {; } \\
& \mathrm{C}_{2} . \quad \text { si } \mathrm{X} \subseteq \mathrm{X} \subseteq \mathrm{A}, \quad \mathrm{C}(\mathrm{X}) \subseteq \mathrm{C}(\mathrm{Y}) \text {; } \\
& \mathrm{c}_{3} \text {, si } \mathrm{x} \subseteq \mathrm{A}, \quad \mathrm{c}(\mathrm{c}(\mathrm{x}))=\mathrm{c}(\mathrm{x}) \text {. }
\end{aligned}
$$

L'operador C s'anomena operador de consequlència.

Realment A.Tarski imposaya dues restriccions : $C_{0} \cdot \operatorname{Card}(\mathrm{A}) \leq \chi_{0}$ 
i $c_{2}^{\prime} . \quad C(x)=\bigcup C(F)$, on $F \subseteq X$ i $F$ és finit (en lloc de (2)). Aquesta definició de A.Tarski, si bé inspirada en el càlcul de proposicions i correcta a l'hora de formalitzar el calcul de proposicions; esdevé massa restrictiva ja que exclou, d'antuvi, les lògiques infinitàries cada co més importants. Hem pogut veure (V.VERDd [1978]) que la lògica probabilistica (en el sentit $\sigma$ ) és una lògica infinitària. .

Hem indicat $j a$, en el cas de les algebres de Hilbert (reticles de Hilbert), que la col.lecció dels sistemes deductius és un sistema clausura $i$ un teorema general de M.WARD [1942] ens diu que un sistema clausura fo $i$ un operador de conseqúència $c$ és el mateix; si fem $b=\{x: c(x)=x\}$ es

$$
c(x)=\bigcap\{\mathrm{Y}: \mathrm{Y} \in \boldsymbol{b} \text { i } \mathrm{Y} \geqslant \mathrm{X}\} \text {. }
$$

Teoremes com el de Schmidt - diu : un operador C es finitari si, i només si, el seu sistema clausura és fortament inductiu - i el de Pierce - diu : si C és finitari, els elements del sistema clausura 6 irreductibles constitueixen una base mínima de $f$ - suggereixen la possibilitat de donar propietats de $C$ a travers del seu sistema clausura associat.

Una questió important que cal resoldre $i$ en la que hem treballat (cE.J.Pla [1976]; V.Verdú [19781) consisteix en imposar co 20 ms a $C$ (a $b$ ) per tal que es verifiqui el teorema de la deducció de Tarski. Ara bé el teorema de la deducció de Tarski és un teorema intimament lligat amb l'operació

$i$ pot ocbrrer que, en $A$, no es disposi de cap operacio. . guines condicions ha de satisfer, doncs, $c(o f)$ per tal de poder introduir en A una operací. que ens permeti d'enunciar el teorema de la deduccio:

$$
y \in C(x, x) \quad s i, i \text { només si, } x, y \in c(x) \text { ? }
$$

Les respostes donades per J.Pla i V.Verdú difereixen en el seglfent sentit : 
mentres que el primer dóna una condició que permet definir, en $A$ directament, una operació que verifica el teorema de la decluccio, el segon fa la segient construccib :

Tot operador $C$ permet de definir una relació d'equivalència indurida per la relació de preordre:

$$
x \leqslant{ }_{C} y \text { si, i només si, } y \in C(\{x\}) .
$$

rodem doncs passar al quocient $A / \Longrightarrow C^{i}$ considerar l'algebra quocient $(A / \equiv C, \bar{C})$, on $\bar{C}$ és

$$
\overline{\mathrm{G}}: \subseteq\left(\mathrm{A} / \mathrm{C}_{\mathrm{C}}\right) \longrightarrow \odot\left(\mathrm{A} / \mathrm{D}_{\mathrm{C}}\right)
$$

$i$ es defineix de forma natural.

Aleshores V.Verdú imposa condicions a $(A, C)$ deforma que, en $(\bar{A}, \bar{C})$, es verifiqui el teorema de la deducció.

L'anterior consideració ens proporciona una Iògica $(A, C)$, una lògica $(\bar{A}, \bar{C})$ i una aplicació compatible amb $C$ i $\bar{C}$; la projecció canònica J .

Sabem que $\pi$ és epijectiva, que $(A, C)$ està generat projectivament per $(\bar{A}, \bar{C}) i$ per $\eta i$ que, per tot $x, y \in A, j(x)=\pi(y)$ si, i. només si, $C(x)=c(y)$.

Aquesta idea és la que recull el treball de D.J.BROWN-R.SUSZKO [1973] de forma general quan introdueix el concepte de morfisme bilògic. Si $\left(A_{1}, C_{1}\right)$ i $\left(A_{2}, C_{2}\right)$ són dues àlgebres abstractes $\mathrm{i} h: \mathrm{A}_{1} \longrightarrow \mathrm{A}_{2}$ és una aplicació que verifica : $\mathbb{B B}_{1}$. h és morfisme (i.e. $\mathrm{h}^{-1}(\mathrm{Y}) \in G_{1}$, per cada $\mathrm{Y} \in \ell_{2}$ );

$\mathrm{MB}_{2} \cdot \quad h$ genera projectivament $\left(A_{1}, C_{1}\right)$ a partir de $\left(A_{2}, C_{2}\right.$ ) i h (i.e. $b_{1}=h^{-1}(Y): y \in b_{2}$ );

$\mathrm{MB}_{3} . \quad \mathrm{h}$ és epijectiva, direm que $h$ es un morfisme bilògic entre $\left(A_{1}, C_{1}\right) \pm\left(A_{2}, C_{2}\right)$. La importància real d'aquesta definició - que justifica el seu nom - és la segllent :

h és un morfisme bilògic entre $\left(A_{1}, C_{1}\right)$ i $\left(A_{2}, C_{2}\right)$ si, i nomes si, 
(1) h és un epimorfisme;

(2) $\equiv_{h} \subseteq \bar{C}_{1}$;

(3) h es una bijecció sobre els tancats;

$$
\text { Es a dir: } \begin{aligned}
h^{-1}(h(x))=x, & x \in \sigma_{1} ; \\
& h\left(h^{-1}(y)\right)=x, \quad y \in \sigma_{2} ;
\end{aligned}
$$

(Aquf convé observar que, si h és un morfisme bilògic, la relació d'equivalència que indueix en $A$ és més petita que la relació d'equivalència natural induida per $C_{1}$.)

Es clara la importància dels morfismes bilògics ja que conserven els tancats (és a dir la lògica).

Aquesta situació permet plantejar de manera natural el segllent tipus de questions: cớm són les lògiques abstractes (A,C) que admeten un morfisme bilògic amb una lògica abstracta ( $\left.A^{\prime}, C^{i}\right)$ booleana, de Heyting, reticular de Hilbert, de Hilbert,...? Quan diem una Iògica booleana, etc. volem dir una lògica en lá que A és una àlgebra de Boole, etc, i $C^{\prime}$ és l'operador de consequència associat al sistema clausura de tots els sistemes deductius de $A^{t}$.) En V.Verdú (op.cit.) es troben respostes en aquesta lfnia $i$ més encara, ja que s'arriba a caracteritzar $(A, C)$ de forma que sigui bilògica amb la lògica $\sigma$-probabilística que és infinitària.

Dialtres questions que es poden plantejar en relacib amb els operadors de consequència són : (i) la questió del nuclis deductius, (2) la questib de les negacions.

La qliesti $\delta$ dels nuclis deductius - introduits per A.Tarski [1930] és 1a seglent : donada una àlgebra abstracta $(A, C)$ hom pot pre-

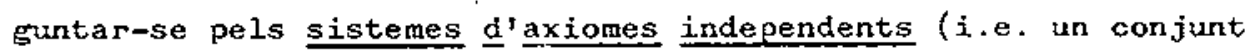
$N \subseteq A$ és un nucli deductiu de $X \subseteq A$ si, $i$ només $s i, C(N)=C(X)$ $i$, si $x \in N, x \notin C(N-\{x\})$.) Es veu que això equival a trobar un conjunt $N$ minimal en la clässe dels conjunts $Y$ amb $C(Y)=C(N)$. Sobre els nuclis deductius podem formular les segulents preguntes : 
- en quines condicions existeixen nuclis deductius? (Hom pot pensar en els espais vectorials $i$ en els A-mòduls, Com podem introduir formalment una propietat que ens faci el paper de teorema de Steinitz?) Tarski (os.cit, demostra que tot $x$ admet una base ordenada (això és una successió $\left(x_{n}\right)_{n \in N}$ tal que

$$
c(x)=c\left(\left\{x_{1}, \ldots, x_{n}, \ldots+f\right) \quad \text { i } \quad x_{n+1} \& c\left(\left\{x_{1}, \ldots, x_{n} t\right)\right)\right.
$$

però una base ordenada no és pas necessàriament un nucli deductiu;

- en quines condicions un conjunt $x$ admet un nucli contingut en $X$ ?

- en quines condicions tots els nuclis tenen el mateix cardinal?

Un primer anàlisi es pot trobar en F.A.Sales-J.Pla [1976].

La quiestió de les negacions lligat amb les lògiques abstractes (A,C) es troba en A.Tarski [1930]; D.J.Brown-R.Suszko [1973]; Rasiowa [1975]; J.Pla [1977]; V.Verdú [1978] i es planteja en els termes segilents :

cóm associar a cada $x \in A$ un element $x^{\prime}$ que, en certa manera, sigui una negación de $x$ ? Si pensem amb una negació d'ordre cal disposar en A d'un ordre $i$, en general, en A no n'hi tenjm cap, però sí que tenim un ordre en $A /=c$. Això ens suggereix passar a 1 quocient $i$ veure $s i$ el que hem definit en A dóna una negació en el quocient.

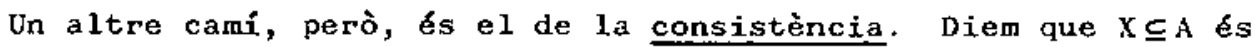
consistent $s i, i$ només $s i, c(x) \neq A$. Aleshores podem donar les seguients definicions :

* diem que $x^{\prime}$ és una negació clàssica de $x$ si, $i$ només si, per cada $\mathrm{X} \subseteq \mathrm{A}, \mathrm{x} \in \mathrm{C}(\mathrm{X}) \mathrm{si}, i$ només $\mathrm{si}, \mathrm{C}\left(\mathrm{X}, \mathrm{x}^{1}\right)=\mathrm{A}$ (Brown-Suszko)

t dien que $x^{\prime}$ és una negació intuicionista de $x$ si, $i$ només si, per cada $x \subseteq A, x^{\dagger} \in C(x)$ si, $i$ nomes si, $C(x, x)=A$ (Rasiowa) 
* diem que $x$ ' és una negació feble de $x$ si, $i$ només si, per

cada $x \subseteq A, C\left(x, x^{\prime}\right)=A$ i $C(x) \cap C\left(x^{\prime}\right)=C(\phi) \quad($ Tarski)

Aquestes són totes les negacions que hom pot definir vinculades a l'operador $\mathrm{C}$ ?

Quines relacions existeixen entre elles? quines jassen a 1 quocient com a negacions d'ordre? ...

Tot el que hem dit fins ara és ja clàssic en el sentit que segueix una línia iniciada els anys 1930 al 1950 . Però recerques pos posteriors han posat de manifest altres implicacions - en el sentit algebraic de la paraula - que no estan gens vinculades amb la implicació de Hilbert i que, per tant, el seu coneixament és mol més vague.

Si pensem en una àlgebra de Boole com en un reticle amb el màxim 1 i minim 0 , distributiu i complementat, podem preguntar-nos qué i com fóra una estructura algebraica que fós :

(1) àlgebra de Boole a menys de la distributivitat;

(2) àlgebra de Boole a menys de la complementació.

Podríem fer un estudi formal d'aquest tipus d'àlgebres, però això podria semblar una disquisició ben poc interessant. No és pas així.

(1) Les àlgebres o reticles ortomodulars.

L'anàlisi de la Mecànica quàntica s'enfronta amb l'àlgebra dels subespais tancats d'un espai de Hilbert. Aquesta algebra constitueix un reticle complementat via $l^{\prime o r}$ togonal que no és distributiu - ni tan sols modular. Ara bé, verifica la llei ortomodular:

$x \leqslant y$ implica $y=x \vee\left(x^{\perp} \wedge y\right)$, per tot $x, y$. (cf.Bodiou (op.cit.), S.S Holland [1966],J.M.JAUCH [1976]). En les àlgebres ortomodulars podem definir una implicacio (en el sentit de Rasiowa, op.cit.) i una operació ordenadora (en el sentit de F.A.Sales [1974]): 


$$
x \rightarrow y=x^{\perp} \vee(x \wedge y)
$$

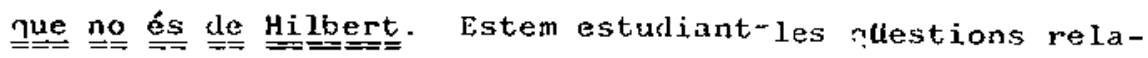
tives a les rejresentacions de stone d'aquest tious it'à gebres.

(2) Els conjunts "difusos" (Fuzzy)

Si $E \neq \neq^{*} \phi$ i $I=[0,1] \subseteq \mathbb{R}$, anomenem classe dels conjunts "difu$\operatorname{sos}^{\prime \prime}$ a

$$
\mathrm{I}^{\mathrm{E}}=\{\mathrm{x}: \mathrm{x}: \mathrm{E} \longrightarrow \mathrm{I}\} \text {. }
$$

Definim $\quad x \vee y=\sup (x, y), \quad x \wedge y=\inf (x, y)$ i $x^{c}=1-x$. Obtenim un reticle amb $\mathrm{c} i \mathrm{i}$, distributiu, que no és comPlementat.

Aquest reticle - $i$ això constitueix un camp totalment verge admet moltes operacions implicatives (i.e. donats dos conjunts lifusos $x, y$, definim

$$
(x \cdot y)_{t}=\left\{\begin{array}{llll}
1 & \text { si } & x_{t} & y_{t} \\
a_{t} & \text { si } & x_{t} & y_{t}
\end{array} ; \text { on } a_{t} \in(0,1) .\right)
$$

Entre les operacions implicatives trobem: l'oneració inplicativa de E. ’OST (que proporciona a $\mathrm{I}^{\mathrm{E}}$ l'estructura d'jlgebra de Heyting es l'únjca operació implicativa classica o de Hilbert possible) i l'operació de J.LUKASIEWTCZ (que rlota a

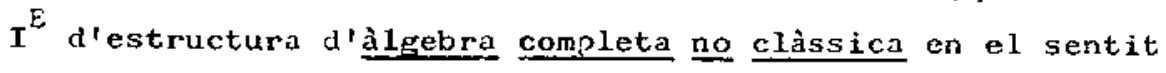
de J.jla [1976] i d'àlgebra de jales, que no és de Abbott i. aqui queda justificada la nomenclatura dbbott-Sales.) En aquest context resta obert el problema de classificar totes les operacions implicatives possibles $i \mathrm{~A}$. TORRENS hi està treballant.

\section{BIBLIOGRAFIA}

ABBOTT, J . C.

(1967) "Semi-boolean algebra", Matematicki Vesnik,4,p.177-198

(1970) "Trend in lattice theory", Van Nostrand. 
BODroU, G.

(1964) "Théorie dialectique des probabilités", Gauthier-Villars. BROWN, D. !SUSZKO, R.

(1973) "Abstracts Logics". Dissert.Math., CII, p.9-40. CURRY, H.

(1952) "Lef̧ons de logique algebraique". Gauthier-Villars. DIEGO,A.

(1965) "Sobre las álgebras de Hilbert" Notas de Lógica Matemática, $\mathrm{n} \cong 12$.

(1966) "Sur les algébres de Hilbert". Gauthier-Villars ESTEVA, $\mathbf{r}$,

(1973) "Negaciones en retfculos". Actas $1^{\text {as }}$ jornadas mat. hispano-1usas, p. 295-302. Lisboa.

(1976) "Contribución al estudio de la estructura de1 conjunto de negaciones definidas en un reticulo". Stochastica, $v . I, n \circ 1$.

HALMOS, P.R.

(1955) "Algebraic Logic I (Monadic Boolean algebras)". Comp.

$$
\text { Math.,12, p.217-249. }
$$

HENKIN, L-MONK, J . D . -TARSKI, A .

(1971) "Cylindric Algebras". North-Holland.

HENKIN, L . -TARSKI, A

(1960) "Cylindrical algebras" Summ.Come11. Univ. vol.III, p.332-340

(1961) "Cylindric algebras" Am.Math.Soc., p. 83-113 HOLLAND, S.S.

(1966) "The current interest in orthomodular lattices". Van Nostrand.

JAUCH , J.M.

(1976) "Logic and probability in Quantum Mechanics". D.Reidel Pub. MONTEIRO,A.

(1957) "Normalidad en las álgebras de Heyting monàdicas". UMA.

(1960) "A lgebras monádicas"- Atas de 2 Colbquio Brasileiro de Matemática, p.33-52.

(1960) "Sur le calcul propositionnel implicatif positif". Curs donat a la U.N.S. 
(1967) "Construction des algèbres de Lukasiewicz trivalents dans les algèbres de Boole monadiques I". Math Japan, v.12, P.1-23.

(1971) "La semi-simplicité des algèbres de Boole topologiques et les sistemes deductifst. UMA, v. 25 .

(1974) "L'arithmétique des filtres et les espaces topologiques 1 i 2 ". UNS, v. 29-30.

PIERCE, R.S.

(1968) "Introduction to the theory of abstract algebras".

New York.

PLA, J.

(1976) "Algebras de Hilbert I". Curs de doctorat. Barcelona.

(1976) "Sobre S y T negaciones' en 16gicas abstractas". Actas $3^{\underline{a}}$ jornadas hispano-lusas de matemáticas Málaga.

(1977) "Algebras de Hilbert $\mathrm{II}^{\mathrm{N}}=$ Curs de doctorat. Barcelona.

(1978) "Negacions en estructures reticulars". En projecte. RASIOWA, H.

(1974) "An algebraic Approach to Non-classica1 Logics". NorthHolland.

RASIOWA, H-SIKORSKI, R.

(1970) "The Mathematics of Metamathematics". Mon. Mat.Warszawa,41. SALES, F de A.

(1971) "Operaciones ordenadoras y álgebras de Hilbert". Reunión anua1 matemáticos españoles. Murcia.

(1973) "Algebras de Hilbert". Curs de doctorat. Barcelona.

(1974). "Sistemas deductivos". Id. Barcelona.

SALES,F de A-PLA, J.

(1976) "Sobre núcleos deductivos". Actas 3a jornadas matemáticas hispano-1usas. Málaga.

TARSKI, A .

(1972) "Logiue, sémantique, methamatématique I". Armand Colin.

(1974) "Iden, II".

VẸRDO, V.

(1978) "Contribució a l'estudi de certs tipus de lògiques abstractes". Tesi doctoral. Barcelona. 
NiRO, M.

(1942) "The closure operators of a lattices". Annals of liath, $43, \mathrm{n} 9_{2}$, p. $191-196$. 